\title{
A Study on the Thermodynamic Consistency of the Park-Paulino-Roesler (PPR) Cohesive Fracture Model
}

\author{
Daniel W. Spring ${ }^{\mathrm{a}}$, Oliver Giraldo-Londoño ${ }^{\mathrm{a}}$, Glaucio H. Paulino ${ }^{\mathrm{a}, \mathrm{b}}$ \\ ${ }^{a}$ Department of Civil and Environmental Engineering, University of Illinois at Urbana-Champaign, Urbana, Il, United States \\ ${ }^{b}$ School of Civil and Environmental Engineering, Georgia Institute of Technology, Atlanta, GA, United States
}

\begin{abstract}
Although the Park-Paulino-Roesler (PPR) potenital-based cohesive zone fracture model was not derived based on a thermodynamics consistency principle, we investigate the thermodynamic consistency of the PPR model under conditions of loading, unloading and reloading. First, we present a general anisotropic Helmholtz free energy function. Then, we reformulate the PPR model into the anisotropic Helmholtz form, and investigate the consistency of the model and the various unloading/reloading relations, which have been proposed for use with the model. By recasting the PPR model into the Helmholtz form, we illustrate that the PPR cohesive potential, while not designed with thermodynamic consistency in mind, is thermodynamically consistent under the pure loading conditions for which it was designed (as expected). We also demonstrate that the unloading/reloading relations, which are commonly used with the PPR model, are not thermodynamically consistent; however, through our investigation, we develop a new coupled unloading/reloading relation, which maintains the thermodynamic consistency of the PPR cohesive model. The considerations addressed in this paper are aimed at achieving a better understanding of the PPR model and other models of similar nature.
\end{abstract}

Keywords: PPR Cohesive Model, Anisotropic Helmholtz Function, Thermodynamic Consistency, Damage Mechanics

\section{Introduction}

Thermodynamically consistent cohesive models are derived from a potential function [1]. There are two primary classes of potential functions. In the first class, the potential is a function of the displacement jump. This form is prevalent in phenomenological cohesive models, and is the class to which the Park-Paulino-Roesler (PPR) cohesive model belongs [2]. Potential functions of this form do not contain a built-in unloading/reloading relation. This is an attractive feature of the model, as any desired feature can be easily incorporated in the basic PPR framework $[3,4]$. However, without the inclusion of $e x$ ternal history parameters and an unloading/reloading relation, these types of models are reversible. The reversibility of the potential function leads to the commonly applied critique that models of this type do not satisfy the second law of thermodynamics (i.e. what is done can be undone) $[1,5,6,7,8]$. Alternatively, some researchers use a thermodynamic potential, specifically a Helmholtz free energy function, to derive a thermodynamically consistent cohesive model $[9,10,11]$. In this class of functions, the potential depends on both the displacement jump and a set of internal variables related to the deformation history. Thus, the unloading/reloading relation is intrinsic to the potential, and all states of loading, unloading and reloading are defined by a single function. In the following discussion, we will

Email addresses: spring2@illinois.edu (Daniel W. Spring), grldlnd2@illinois . edu (Oliver Giraldo-Londoño),

paulino@gatech. edu (Glaucio H. Paulino) recast the PPR model into the Helmholtz form, to illustrate that the PPR cohesive potential, while not designed with thermodynamic consistency in mind, is thermodynamically consistent under the pure loading conditions for which it was designed. We will also demonstrate that the unloading/reloading relations, which are commonly used with the PPR model, are not thermodynamically consistent; however, we can extract a new unloading/reloading relation from the Helmholtz form which is thermodynamically consistent.

The remainder of the paper is organized as follows. In the next section, we present a general form for the anisotropic Helmholtz free energy function. In Section 3, we reformulate the PPR potential function as an anisotropic Helmholtz function. In Section 4, we investigate the thermodynamic consistency of the PPR cohesive model under conditions of loading and unloading/reloading. Based on our investigation, we develop a new coupled unloading/reloading relation in Section 5; which maintains the thermodynamic consistency of the PPR cohesive model. In Section 6, we present an example of an interface undergoing mixed-mode loading and unloading; which highlights the significance of the new formulation. Finally, we provide some concluding remarks in Section 7.

\section{The Anisotropic Helmholtz Free Energy Function}

Here, we outline a framework for generating a thermodynamically consistent constitutive relation for inelastic materials [12]. We consider the inelasticity to be associated with stiffness

May 16, 2016 
degradation of the material, and thus work within the framework of damage mechanics, i.e., damage is herein defined as a reduction in the material's secant stiffness [13]. For most generality, only a couple of assumptions are made. First, following the frequently applied assumption in stress-strain based constitutive models $[14,15]$, we assume a decomposition of the potential energy into shear and normal components. Second, we assume that damage may be captured by a set of scalar-valued damage parameters [1]. With these two assumptions, the general Helmholtz free energy function, $\Psi$, for anisotropic materials, takes the following form:

$$
\Psi=\sum_{i=1}^{n}\left(1-d_{i}\right) \Psi_{i}=\left(1-d_{n}\right) \Psi_{n}+\left(1-d_{t}\right) \Psi_{t},
$$

where $\Psi_{n}$ is the energy related to normal separation and $\Psi_{t}$ is the energy related to tangential (or shear) separation. Moreover, $d_{n}$ and $d_{t}$ are scalar damage parameters related to the evolution of damage in the normal and shear directions, respectively. The scalar damage parameters are continuous and take values between 0 and 1 (i.e. $d_{n} \in[0,1]$ and $d_{t} \in[0,1]$ ) where 0 indicates no damage, and 1 indicates complete damage. In a related investigation, Mosler and Scheider [1] included a third assumption: that the different damage mechanisms in the normal and shear directions are multiplicatively coupled. Thus, they proposed the following form for the anisotropic Helmholtz free energy function:

$$
\Psi=\sum_{i=1}^{n} \prod_{j=1}^{n}\left(1-d_{i}^{(j)}\right) \Psi_{i}
$$

However, we note that this is a subset of the more general form (1), where:

$$
\left(1-d_{n}\right)=\left(1-d_{n}^{(n)}\right)\left(1-d_{n}^{(t)}\right),\left(1-d_{t}\right)=\left(1-d_{t}^{(n)}\right)\left(1-d_{t}^{(t)}\right) .
$$

In both cases, the effective damage parameters are continuous and vary between 0 and 1 .

In the context of a purely mechanical theory, we can use the classical Coleman and Noll procedure [16] to derive a thermodynamically consistent, anisotropic constitutive relation. In this setting, the Clausius-Duhem dissipation inequality reads:

$$
D=\stackrel{\circ}{w}-\dot{\Psi} \geq 0
$$

where $\stackrel{w}{w}$ is the stress power and $\dot{\Psi}$ is the time derivative of the Helmholtz free energy function. In the current context of cohesive zone models, the stress power is written as [1]:

$$
\stackrel{\circ}{W}=\boldsymbol{T} \cdot \llbracket \dot{\boldsymbol{u}} \rrbracket,
$$

where $\boldsymbol{T}$ is the traction vector, and $\llbracket \dot{\boldsymbol{u}} \rrbracket$ is the time derivative of the displacement jump. Inserting the expressions for the stress power and the time derivative of the general Helmholtz free energy function (1) into the dissipation inequality (4), we obtain
1

$$
\begin{aligned}
D=\stackrel{\circ}{W}-\dot{\Psi}=\boldsymbol{T} \cdot \llbracket \dot{\boldsymbol{u}} \rrbracket & -\left(1-d_{n}\right) \frac{\partial \Psi_{n}}{\partial \llbracket \boldsymbol{u} \rrbracket} \cdot \llbracket \dot{\boldsymbol{u}} \rrbracket+\Psi_{n} \dot{d}_{n} \\
& -\left(1-d_{t}\right) \frac{\partial \Psi_{t}}{\partial \llbracket \boldsymbol{u} \rrbracket} \cdot \llbracket \dot{\boldsymbol{u}} \rrbracket+\Psi_{t} \dot{d}_{t} \geq 0 .
\end{aligned}
$$

From the above expression, we can define the following relations for the cohesive tractions:

$$
T_{n}=\left(1-d_{n}\right) \frac{\partial \Psi_{n}}{\partial \llbracket \boldsymbol{u} \rrbracket}, \text { and } T_{t}=\left(1-d_{t}\right) \frac{\partial \Psi_{t}}{\partial \llbracket \boldsymbol{u} \rrbracket} ;
$$

which results in the following form for the dissipation inequality:

$$
D=\Psi_{n} \dot{d}_{n}+\Psi_{t} \dot{d}_{t} \geq 0 .
$$

Since the elastic energies are assumed to be non-negative, the dissipation inequality, and thus the second law of thermodynamics [16], is automatically satisfied if the damage parameters are monotonically increasing:

$$
\dot{d}_{n} \geq 0 \quad \text { and } \quad \dot{d}_{t} \geq 0 .
$$

Therefore, in order for an anisotropic constitutive relation to be thermodynamically consistent, the above constraints need to be met.

\section{Reformulating the PPR Potential as an Anisotropic Helmholtz Function}

To demonstrate the thermodynamic consistency of the PPR potential function [2], under the pure loading conditions it was designed for, we recast the potential function into the form of the Helmholtz free energy function (1). With some algebraic maneuvering, the energies, $\Psi_{n}$ and $\Psi_{t}$, consistent with the PPR model, take the form:

$$
\Psi_{n}=\frac{1}{2} E_{n} \Delta_{n}^{2} \quad \text { and } \quad \Psi_{t}=\frac{1}{2} E_{t} \Delta_{t}^{2},
$$

where $\Delta_{n}$ and $\Delta_{t}$ are the normal and tangential crack opening widths, respectively. The variables $E_{n}$ and $E_{t}$ shown above are initial stiffness parameters in the normal and tangential directions, respectively. These parameters are consistent with the original PPR cohesive model proposed by Park et al. [2] (see Appendix A), and are defined as:

$$
\begin{aligned}
& E_{n}=-\frac{\Gamma_{n}}{\delta_{n}^{2}}\left(\frac{m}{\alpha}\right)^{m-1}(m+\alpha)\left[\Gamma_{t}\left(\frac{n}{\beta}\right)^{n}+\left\langle\phi_{t}-\phi_{n}\right\rangle\right], \\
& E_{t}=-\frac{\Gamma_{t}}{\delta_{t}^{2}}\left(\frac{n}{\beta}\right)^{n-1}(n+\beta)\left[\Gamma_{n}\left(\frac{m}{\alpha}\right)^{m}+\left\langle\phi_{n}-\phi_{t}\right\rangle\right],
\end{aligned}
$$

\footnotetext{
${ }^{1}$ We assume here, for simplicity, that the Helmholtz free energy is not a function of any structural tensor. In the remainder of the paper, we consider the crack faces to be parallel, limiting our investigation to the constitutive component of the model and eliminating the need to include energetically consistent conjugate stresses [1]
} 
where $\delta_{n}$ and $\delta_{t}$ are the final crack opening widths in the normal and tangential directions:

$$
\begin{gathered}
\delta_{n}=\frac{\phi_{n}}{\sigma_{\max }} \alpha \lambda_{n}\left(1-\lambda_{n}\right)^{\alpha-1}\left(\frac{\alpha}{m}+1\right)\left(\frac{\alpha}{m} \lambda_{n}+1\right)^{m-1}, \\
\delta_{t}=\frac{\phi_{t}}{\tau_{\max }} \beta \lambda_{t}\left(1-\lambda_{t}\right)^{\beta-1}\left(\frac{\beta}{n}+1\right)\left(\frac{\beta}{n} \lambda_{t}+1\right)^{n-1} .
\end{gathered}
$$

Moreover, $\alpha$ and $\beta$ are shape parameters which control the softening shape of the traction-separation relation in the normal and shear directions, respectively. Both $\alpha$ and $\beta$ are $\geq 1$. If $\alpha$ (or $\beta$ ) is set equal to 2 , the softening relation is almost linear, whereas if $\alpha$ (or $\beta$ ) is less than or greater than 2, the relation is concave or convex, respectively. Moreover, the non-dimensional exponents, $m$ and $n$, are evaluated from the shape parameters and the initial slope indicators $\left(\lambda_{n}, \lambda_{t}\right)$ :

$$
m=\frac{\alpha(\alpha-1) \lambda_{n}^{2}}{\left(1-\alpha \lambda_{n}^{2}\right)}, \quad n=\frac{\beta(\beta-1) \lambda_{t}^{2}}{\left(1-\beta \lambda_{t}^{2}\right)}
$$

where the initial slope indicators relate the final crack opening widths to the crack opening widths at peak cohesive stress. The energy constants $\Gamma_{n}$ and $\Gamma_{t}$ are related to the normal and tangential fracture energies [2]. When the normal and tangential fracture energies are different $\left(\phi_{n} \neq \phi_{t}\right)$, the energy constants are:

$$
\Gamma_{n}=\left(-\phi_{n}\right)^{\left\langle\phi_{n}-\phi_{t}\right\rangle /\left(\phi_{n}-\phi_{t}\right)}\left(\frac{\alpha}{m}\right)^{m}, \quad \Gamma_{t}=\left(-\phi_{t}\right)^{\left\langle\phi_{t}-\phi_{n}\right\rangle /\left(\phi_{t}-\phi_{n}\right)}\left(\frac{\beta}{n}\right)^{n},
$$

where the Macaulay bracket $\langle\cdot\rangle$ is defined such that $\langle x\rangle=$ $(|x|+x) / 2$. When the normal and tangential fracture energies are equal $\left(\phi_{n}=\phi_{t}\right)$, the energy constants are:

$$
\Gamma_{n}=-\phi_{n}\left(\frac{\alpha}{m}\right)^{m}, \quad \Gamma_{t}=\left(\frac{\beta}{n}\right)^{n}
$$

The damage parameters consistent with the PPR model are functions of two internal history parameters $\left(\kappa_{n}, \kappa_{t}\right)$ [12], and take the form:

$$
\begin{aligned}
& d_{n}\left(\kappa_{n}, \kappa_{t}\right)=1-\frac{T_{n}^{\prime}\left(\kappa_{n}, \kappa_{t}\right)}{E_{n} \kappa_{n}}, \\
& d_{t}\left(\kappa_{n}, \kappa_{t}\right)=1-\frac{T_{t}^{\prime}\left(\kappa_{n}, \kappa_{t}\right)}{E_{t} \kappa_{t}},
\end{aligned}
$$

where

$$
\begin{aligned}
T_{n}^{\prime}\left(\kappa_{n}, \kappa_{t}\right)=\frac{\Gamma_{n}}{\delta_{n}} & {\left[m\left(1-\frac{\kappa_{n}}{\delta_{n}}\right)^{\alpha}\left(\frac{m}{\alpha}+\frac{\kappa_{n}}{\delta_{n}}\right)^{m-1}\right.} \\
& \left.-\alpha\left(1-\frac{\kappa_{n}}{\delta_{n}}\right)^{\alpha-1}\left(\frac{m}{\alpha}+\frac{\kappa_{n}}{\delta_{n}}\right)^{m}\right] \\
& \times\left[\Gamma_{t}\left(1-\frac{\kappa_{t}}{\delta_{t}}\right)^{\beta}\left(\frac{n}{\beta}+\frac{\kappa_{t}}{\delta_{t}}\right)^{n}+\left\langle\phi_{t}-\phi_{n}\right\rangle\right],
\end{aligned}
$$

$$
\begin{aligned}
& T_{t}^{\prime}\left(\kappa_{n}, \kappa_{t}\right)=\frac{\Gamma_{t}}{\delta_{t}}\left[n\left(1-\frac{\kappa_{t}}{\delta_{t}}\right)^{\beta}\left(\frac{n}{\beta}+\frac{\kappa_{t}}{\delta_{t}}\right)^{n-1}\right. \\
&\left.-\beta\left(1-\frac{\kappa_{t}}{\delta_{t}}\right)^{\beta-1}\left(\frac{n}{\beta}+\frac{\kappa_{t}}{\delta_{t}}\right)^{n}\right] \\
& \times {\left[\Gamma_{n}\left(1-\frac{\kappa_{n}}{\delta_{n}}\right)^{\alpha}\left(\frac{m}{\alpha}+\frac{\kappa_{n}}{\delta_{n}}\right)^{m}+\left\langle\phi_{n}-\phi_{t}\right\rangle\right] . }
\end{aligned}
$$

Substituting Equations (10), (18) and (19) into Equation (7), the cohesive tractions can be compactly written as:

$$
T_{n}=T_{n}^{\prime}\left(\kappa_{n}, \kappa_{t}\right) \frac{\Delta_{n}}{\kappa_{n}}, \text { and } T_{t}=T_{t}^{\prime}\left(\kappa_{n}, \kappa_{t}\right) \frac{\Delta_{t}}{\kappa_{t}} ;
$$

The internal history parameters $\kappa_{n}$ and $\kappa_{t}$ represent the maximum normal opening and absolute tangential opening in the history of loading, respectively. Because the damage parameters are constrained to be irreversible in Equation (9), to satisfy the dissipation inequality in Equation (4), the history parameter must also be irreversible, i.e.:

$$
\kappa_{n}=\max \left\{\Delta_{n}\right\} \quad \text { and } \quad \kappa_{t}=\max \left\{\left|\Delta_{t}\right|\right\} .
$$

In a numerical setting, these parameters satisfy the following relations from one increment $(i)$ to the next $(i+1)$ :

$$
\kappa_{n}^{i+1}=\max \left\{\kappa_{n}^{i}, \Delta_{n}^{i+1}\right\} \quad \text { and } \quad \kappa_{t}^{i+1}=\max \left\{\kappa_{t}^{i},\left|\Delta_{t}^{i+1}\right|\right\} .
$$

Therefore, when unloading occurs, i.e., $\kappa_{n}^{i+1}=\kappa_{n}^{i}$ and $\kappa_{t}^{i+1}=$ $\kappa_{t}^{i}$, the corresponding damage parameter does not decrease. In the pure loading scenario, $\kappa_{n}^{i+1}=\Delta_{n}^{i+1}$ and $\kappa_{t}^{i+1}=\left|\Delta_{t}^{i+1}\right|$; and the tractions resulting from the Helmholtz free energy function are equivalent to those from the PPR potential function (see Appendix A).

There are three distinct scenarios where unloading occurs, as illustrated in Figure 1. In order for an unloading/reloading relation to be thermodynamically consistent, the damage parameters during unloading need to satisfy the constraints in Equation (9) in all three scenarios. In the following section, we will plot the evolution of the damage parameters for the PPR model under conditions of loading and unloading/reloading. In the case of unloading/reloading, we will consider both the coupled and uncoupled relations proposed for use with the PPR model [17].

\section{Assessing the Thermodynamic Consistency of the PPR Cohesive Model}

As discussed previously, the primary critique of potentialbased models which only depend on the displacement jump is the reversibility of the model without the inclusion of external history parameters. Thus, it is not surprising that, in conditions of pure loading, cohesive models of this type are indeed thermodynamically consistent (i.e. damage monotonically increases in the model). To show this, we plot a typical traction-separation relation derived from the Helmholtz form of the PPR model, and the corresponding evolution of damage parameters in Figure 2. As illustrated, each damage parameter begins at 0 and monotonically increase to 1 as the separation $\left(\Delta_{n}, \Delta_{t}\right)$ increases 


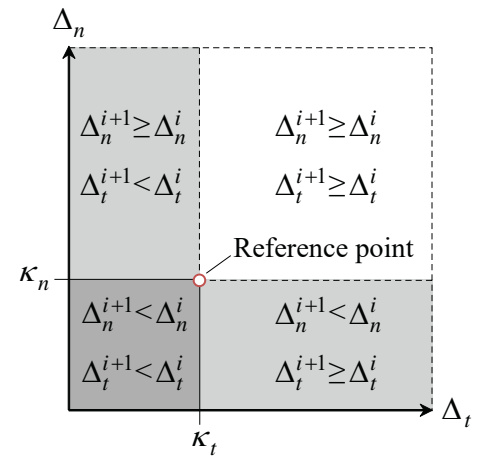

Figure 1: At each state (reference point) of the loading history, there are four possible scenarios for loading to progress. The pure loading condition constitutes the white region $\left(\Delta_{n}^{i+1} \geq \Delta_{n}^{i}\right.$ and $\left.\Delta_{t}^{i+1} \geq \Delta_{t}^{i}\right)$; partial unloading constitutes the two light gray regions $\left(\Delta_{n}^{i+1}<\Delta_{n}^{i}\right.$ and $\Delta_{t}^{i+1} \geq \Delta_{t}^{i}$; or $\Delta_{n}^{i+1} \geq \Delta_{n}^{i}$ and $\left.\Delta_{t}^{i+1}<\Delta_{t}^{i}\right)$; and pure unloading constitutes the dark gray region $\left(\Delta_{n}^{i+1}<\Delta_{n}^{i}\right.$ and $\Delta_{t}^{i+1}<\Delta_{t}^{i}$ ). The reference point corresponds to the point of intersection between the historical maximum normal separation and the historical maximum tangential separation.

to the final crack opening width $\left(\delta_{n}, \delta_{t}\right)$. If this were not the case (i.e. the damage parameter, and thus the damage in the material, decreased), it would indicate that the material has self-healed; a behavior not accounted for in the original formulation of the PPR cohesive model.

Since the unloading/reloading relations which have been proposed for use with the PPR model are both phenomenological and independent of the original potential that it is based upon, their thermodynamic consistency is not guaranteed (as expected). Here, we examine both the uncoupled and coupled unloading/reloading relations [17] and compute their effective damage parameters, to demonstrate the issues inherent in their formulation. A summary of the coupled and uncoupled unloading/reloading relations is listed in Table 1. First, in the uncoupled case, unloading in the normal direction is viewed independently from that in the tangential direction. Typical uncoupled unloading tractions are illustrated in Figures 3(a) and 3(b). Because this unloading relation is not extracted from the cohesive model itself, there is no explicit form assumed for the damage parameters; however, we can compute the effective damage parameters numerically, as illustrated in Figures 3(c) and 3(d).

In Figure 3(c), the normal damage parameter monotonically increases with normal separation. However, when simultaneous unloading in the normal and tangential directions occurs (the pure unloading scenario in Figure 1), the damage parameter decreases with decreasing tangential separation. This is not a physically realistic response. In the absence of self-healing, the damage parameters should not decrease as the material unloads. Similarly, the tangential damage parameter monotonically increases with tangential separation, as illustrated in Figure $3(\mathrm{~d})$, but decreases with normal separation when simultaneous unloading occurs in both directions.

Finally, for the coupled unloading/reloading case, unloading in the normal direction is viewed as dependent on that in the tangential direction. Typical coupled unloading tractions are illustrated in Figures 4(a) and 4(b). This unloading relation is also phenomenological, and not extracted from the cohesive model itself (i.e. there is no explicit form assumed for the damage parameters), thus we compute the effective damage parameters numerically, and illustrate them in Figures 4(c) and 4(d). From the figures, it is clear that neither the normal damage parameter nor the tangential damage parameter demonstrate monotonicity in the region of unloading.

In a thermodynamically consistent model, we would expect each of the damage parameters to display monotonically increasing behavior, regardless of the unloading condition. It is well-known that this behavior is not possible with an uncoupled unloading/reloading relation, thus the unloading/reloading relation must be coupled. In the following section, we derive a new, thermodynamically consistent, coupled unloading/reloading relation for use with the PPR cohesive model.

\section{A Thermodynamically Consistent Unloading/Reloading Relation}

In the previous section, we verified that neither the coupled nor the uncoupled unloading/reloading relations, which have been proposed for use with the PPR cohesive model, are thermodynamically consistent (as expected). However, since the anisotropic Helmholtz form of the PPR model describes all conditions of loading, it contains an intrinsic unloading/reloading relation. The corresponding thermodynamically consistent relations, extracted from the Helmholtz form of the PPR model, are depicted in Figure 5. The damage parameters, equivalent to those computed using Equations (18) and (19), are illustrated in Figures 5(c) and 5(d). As shown, both the normal and tangential damage parameters monotonically increase under both normal and tangential separation. When unloading/reloading occurs simultaneously in both directions, both damage parameters remain constant; which is the only thermodynamically consistent form of these parameters in this scenario (when self-healing is neglected), as per the dissipation inequality (8).

In addition to the cohesive traction vectors in Equation (22), we outline the form of the material tangent stiffness matrix for the thermodynamically consistent unloading/reloading relation:

$$
\mathbf{D}^{v}\left(\Delta_{n}, \Delta_{t}\right)=\left[\begin{array}{ll}
D_{n n}^{v} & D_{n t}^{v} \\
D_{t n}^{v} & D_{t t}^{v}
\end{array}\right]=\left[\begin{array}{cc}
\partial T_{n}^{v} / \partial \Delta_{n} & \partial T_{n}^{v} / \partial \Delta_{t} \\
\partial T_{t}^{v} / \partial \Delta_{n} & \partial T_{t}^{v} / \partial \Delta_{t}
\end{array}\right]
$$

For each of the three distinct unloading scenarios, illustrated in Figure 1, the components $\left(D_{n n}^{v}, D_{n t}^{v}, D_{t n}^{v}\right.$, and $\left.D_{t t}^{v}\right)$ of the tangent matrix become:

$$
\begin{aligned}
\text { 1) If } \Delta_{n}<\kappa_{n} \text { and }\left|\Delta_{t}\right|<\kappa_{t} & \\
D_{n n}^{v}=T_{n}^{\prime}\left(\kappa_{n}, \kappa_{t}\right) \frac{1}{\kappa_{n}} & D_{t t}^{v}=T_{t}^{\prime}\left(\kappa_{n}, \kappa_{t}\right) \frac{1}{\kappa_{t}} \\
D_{t n}^{v}=0 & D_{n t}^{v}=0
\end{aligned}
$$




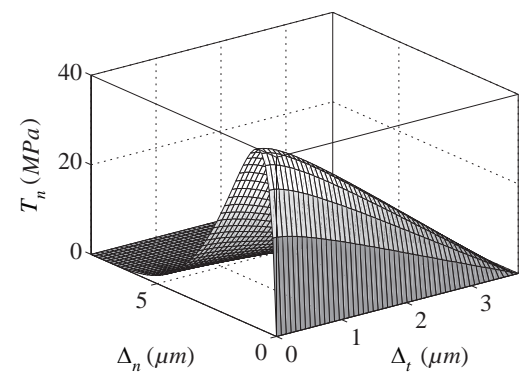

(a)

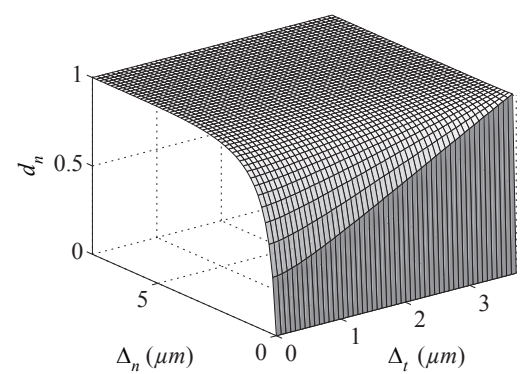

(c)



(b)

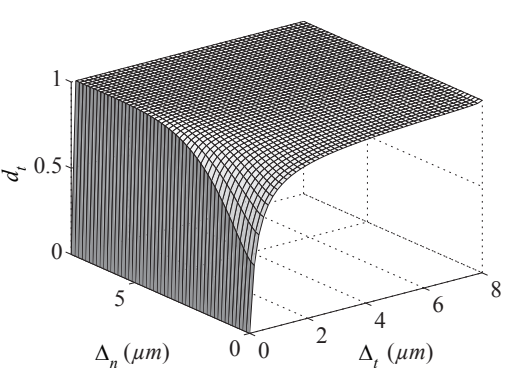

(d)

Figure 2: Depiction of typical traction-separation relations derived from the Helmholtz form of the PPR cohesive model, and the corresponding normal and tangential damage parameters. Traction-separation relations for (a) normal opening and (b) tangential opening. Evolution of the damage parameters for (c) normal opening and (d) tangential opening. The cohesive parameters are: $\phi_{n}=100 \mathrm{~N} / \mathrm{m}, \phi_{t}=200 \mathrm{~N} / \mathrm{m}, \sigma_{\max }=40 \mathrm{MPa}, \tau_{\max }=30 \mathrm{MPa}, \alpha=5, \beta=1.3, \lambda_{n}=0.1$, and $\lambda_{t}=0.2$.



(a)

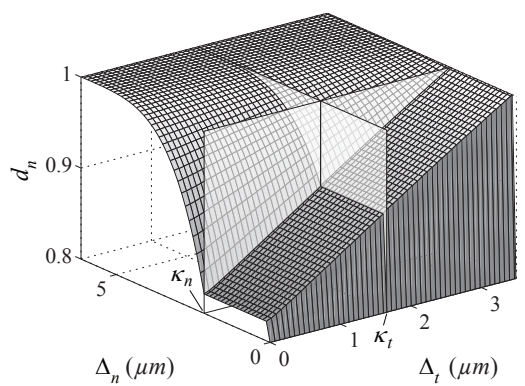

(c)

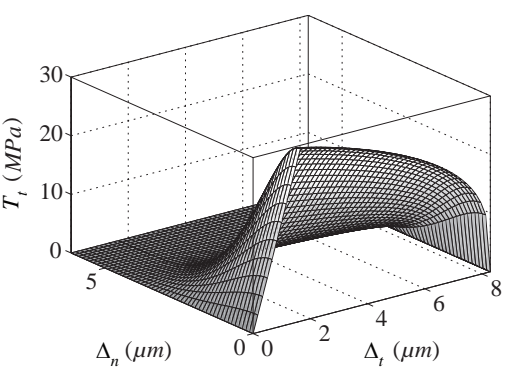

(b)

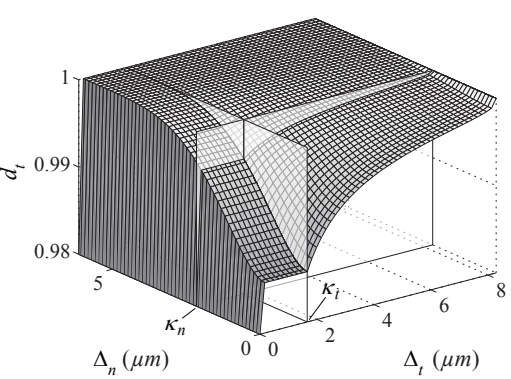

(d)

Figure 3: Depiction of the uncoupled unloading/reloading relation for the PPR cohesive model: (a) traction in the normal direction; (b) traction in the tangential direction; (c) effective damage parameter in the normal direction; (d) effective damage parameter in the tangential direction. The cohesive parameters are: $\phi_{n}=$ $100 \mathrm{~N} / \mathrm{m}, \phi_{t}=200 \mathrm{~N} / \mathrm{m}, \sigma_{\max }=40 \mathrm{MPa}, \tau_{\max }=30 \mathrm{MPa}, \alpha=5, \beta=1.3, \alpha_{v}=1, \beta_{v}=1, \lambda_{n}=0.2, \lambda_{t}=0.1, \Delta_{n_{\max }}=0.35 \delta_{n}$, and $\Delta_{t_{\max }}=0.2 \delta_{t}$.

2) If $\Delta_{n}<\kappa_{n}$ and $\left|\Delta_{t}\right|=\kappa_{t}$

$$
\begin{array}{rlrl}
D_{n n}^{v} & =T_{n}^{\prime}\left(\kappa_{n}, \Delta_{t}\right) \frac{1}{\kappa_{n}} & D_{n t}^{v}=D_{n t}\left(\kappa_{n}, \Delta_{t}\right)\left(\frac{\Delta_{n}}{\kappa_{n}}\right) \\
D_{t t}^{v}=D_{t t}\left(\kappa_{n}, \Delta_{t}\right) & D_{t n}^{v}=0
\end{array}
$$

3) If $\Delta_{n}=\kappa_{n}$ and $\left|\Delta_{t}\right|<\kappa_{t}$

$$
\begin{aligned}
D_{n n}^{v} & =D_{n n}\left(\Delta_{n}, \kappa_{t}\right) & D_{n t}^{v} & =0 \\
D_{t t}^{v} & =T_{t}^{\prime}\left(\Delta_{n}, \kappa_{t}\right) \frac{1}{\kappa_{t}} & D_{t n}^{v} & =D_{t n}\left(\Delta_{n}, \kappa_{t}\right)\left(\frac{\Delta_{t}}{\kappa_{t}}\right)
\end{aligned}
$$


Table 1: Summary of the coupled, uncoupled and thermodynamically consistent unloading/reloading relations for the PPR cohesive model.

Coupled [17]

Uncoupled [17]

Thermodynamically

Consistent

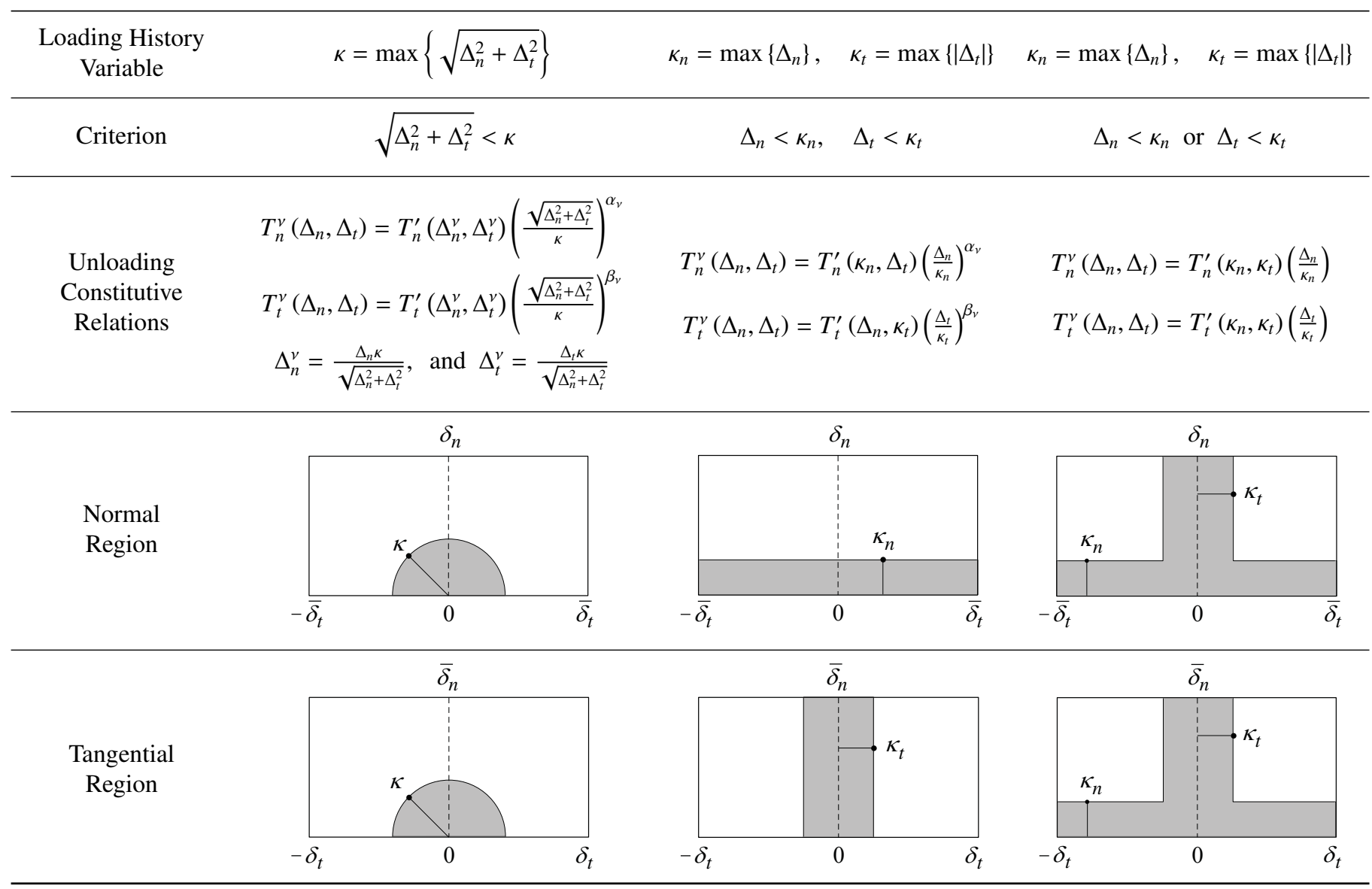

where $D_{n t}\left(\Delta_{n}, \Delta_{t}\right)=\partial T_{n}^{\prime} / \partial \Delta_{t}$ and $D_{t n}\left(\Delta_{n}, \Delta_{t}\right)=\partial T_{t}^{\prime} / \partial \Delta_{n}[18$, 19].

\section{Example: Mixed-Mode Sinusoidal Loading and Unload- ing}

In this section, we evaluate the response of a cohesive interface during nontrivial mixed-mode loading and unloading. The crack faces are assumed to remain parallel to one another, however, the crack opening in the normal direction is assumed to follow a relation independent of that in the tangential direction. The respective opening separations are assumed to follow the relations:

$$
\Delta_{n}=2 \sin (0.15 \tau) \quad \text { and } \quad \Delta_{t}=3.5 \sin (0.12 \tau),
$$

where $\tau$ is a time-like parameter $(0 \leq \tau \leq 100)$, as illustrated in Figures 6 and 7. The response of the interface is investigated for each of the three unloading/reloading relations summarized in Table 1. The cohesive parameters are selected as: $\phi_{n}=\phi_{t}=$ $100 \mathrm{~N} / \mathrm{m}, \sigma_{\max }=40 \mathrm{MPa}, \tau_{\max }=15 \mathrm{MPa}, \lambda_{n}=0.1, \lambda_{t}=0.2$, $\alpha=5, \beta=1.3, \alpha_{v}=1$, and $\beta_{v}=1$. As noted previously, during unloading/reloading of the interface, damage is expected to remain constant, and thus, the slope of the traction-separation relation is also expected to remain constant.



Figure 6: Sinusoidal loading relation in the normal crack opening direction. The following portions of the loading history are delineated: loading (A-B), unloading (B-C), contact (C-D), and reloading (D-E).

For each unloading/reloading relation, the computed normal and tangential traction-separation relations are illustrated in Figures 8 and 9, respectively. From the results, it is clear that the only unloading/reloading relation able to capture the expected response is the one derived from the thermodynamically consistent formulation. The coupled and uncoupled unloading/reloading relations produce significant deviations from the expected results, due to non-monotonic damage accumula- 




(a)

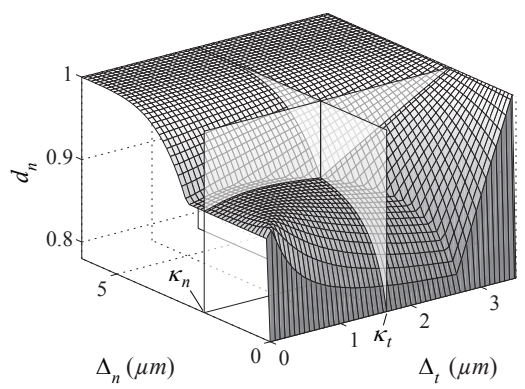

(c)

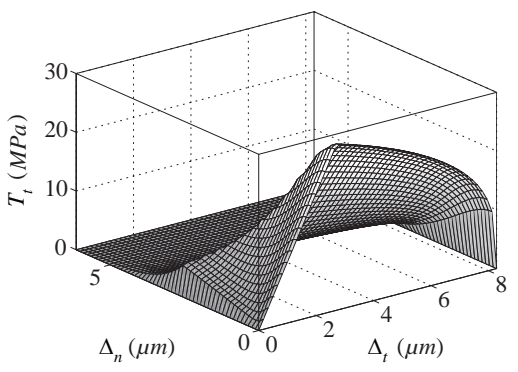

(b)

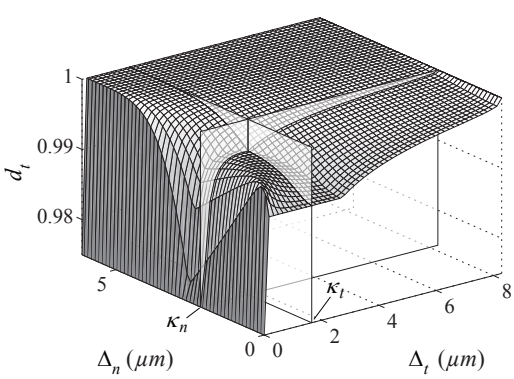

(d)

Figure 4: Depiction of the coupled unloading/reloading relation for the PPR cohesive model: (a) traction in the normal direction; (b) traction in the tangential direction; (c) effective damage parameter in the normal direction; (d) effective damage parameter in the tangential direction. The cohesive parameters are: $\phi_{n}=$ $100 \mathrm{~N} / \mathrm{m}, \phi_{t}=200 \mathrm{~N} / \mathrm{m}, \sigma_{\max }=40 \mathrm{MPa}, \tau_{\max }=30 \mathrm{MPa}, \alpha=5, \beta=1.3, \alpha_{v}=1, \beta_{v}=1, \lambda_{n}=0.2, \lambda_{t}=0.1, \Delta_{n_{\max }}=0.35 \delta_{n}$, and $\Delta_{t_{\max }}=0.2 \delta_{t}$.

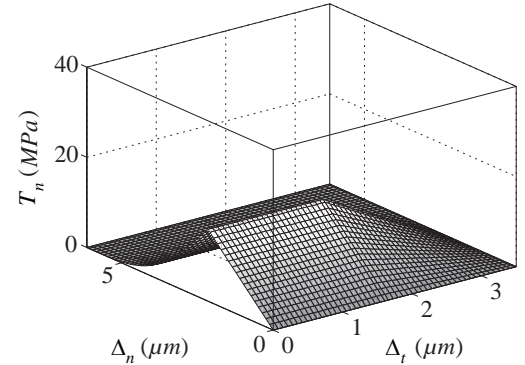

(a)



(c)

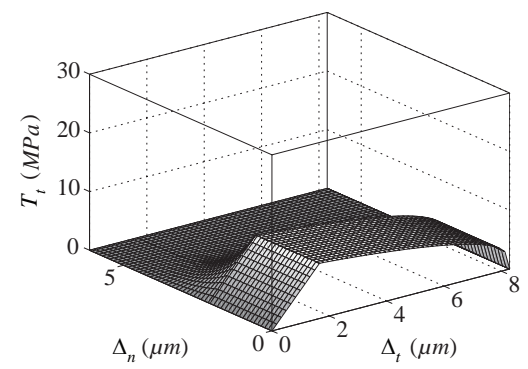

(b)

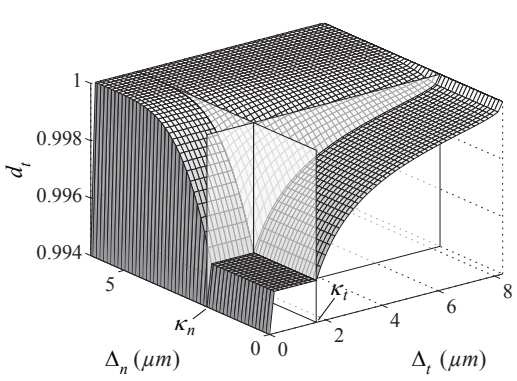

(d)

Figure 5: Depiction of the thermodynamically consistent unloading/reloading relation for the PPR cohesive model, derived from the Helmholtz form of the model: (a) traction in the normal direction; (b) traction in the tangential direction; (c) damage parameter in the normal direction; and (d) damage parameter in the tangential direction. The cohesive parameters are: $\phi_{n}=100 \mathrm{~N} / \mathrm{m}, \phi_{t}=200 \mathrm{~N} / \mathrm{m}, \sigma_{\max }=40 \mathrm{MPa}, \tau_{\max }=30 \mathrm{MPa}, \alpha=5, \beta=1.3, \lambda_{n}=0.2, \lambda_{t}=0.1, \Delta_{n_{\max }}=0.35 \delta_{n}$, and $\Delta_{t_{\max }}=0.2 \delta_{t}$.

tion in these relations. Note that, even though the violation of monotonicity is small (see Figures 3 and 4), the impact of the violation on the global response is large.

The unexpected unloading/reloading behavior, observed us- ing both the uncoupled and coupled relations, could lead to incorrect global behavior if one were to use these relations for problems which experience a significant amount of mixedmode crack face unloading. One prominent example of this is 


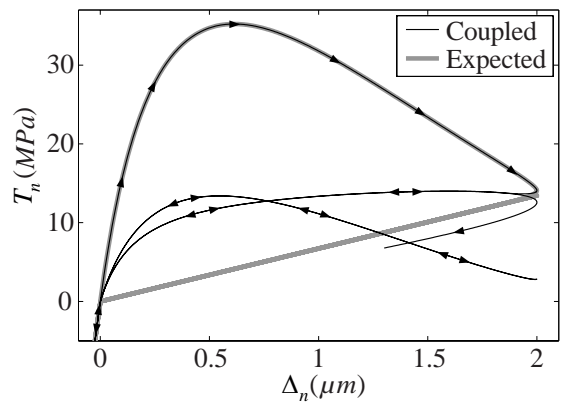

(a)



(b)

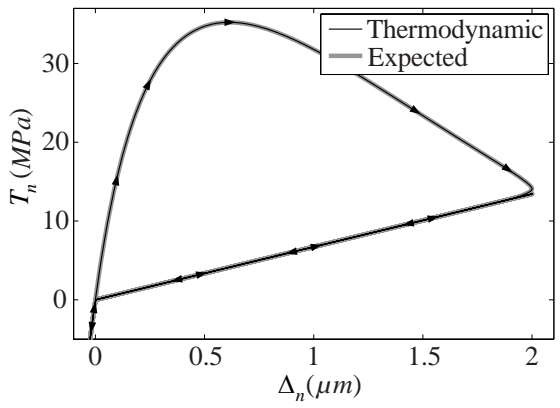

(c)

Figure 8: Tractions in the normal direction during mixed-mode sinusoidal loading/unloading. Computed for the: (a) coupled, (b) uncoupled, and (c) thermodynamically consistent unloading/reloading relations.



(a)

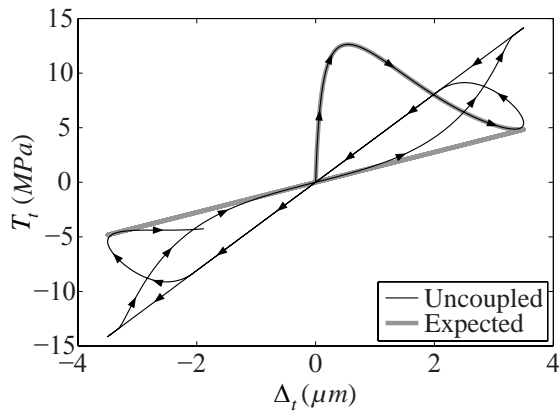

(b)



(c)

Figure 9: Tractions in the tangential direction during mixed-mode sinusoidal loading/unloading. Computed for the: (a) coupled, (b) uncoupled, and (c) thermodynamically consistent unloading/reloading relations.

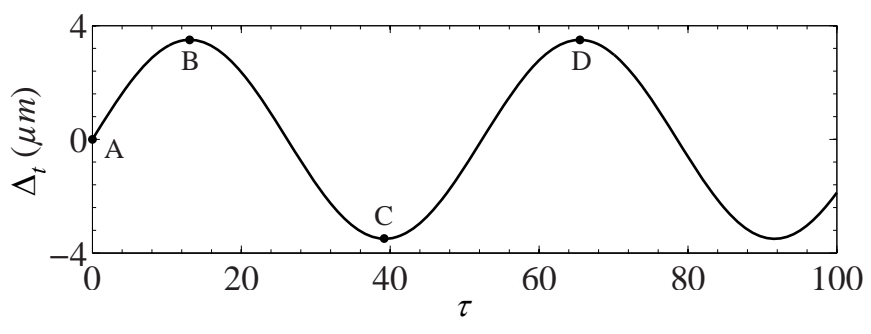

Figure 7: Sinusoidal loading relation in the tangential crack opening direction. The following portions of the loading history are delineated: loading (A-B), unloading (B-C), and reloading (C-D).

in the case of fatigue damage, wherein the cohesive zone experiences repeated cycles of loading, unloading and reloading. Fatigue damage has been modeled with the cohesive zone concept, and is generally introduced through unloading/reloading relations [20, 21, 22]. The aforementioned thermodynamically consistent unloading/reloading relation might be a viable alternative to address fatigue modeling.

\section{Concluding Remarks}

This paper addresses the thermodynamic consistency of the PPR potential-based cohesive fracture model. We demonstrate that the PPR model, while not designed with thermodynamic consistency in mind, is thermodynamically consistent under the pure loading conditions for which it was designed. We do this by proposing a general form for the anisotropic Helmholtz free energy function, and then by recasting the PPR cohesive model to this form. Through the reformulation of the PPR model, we are able to demonstrate that damage accumulates in the model in an irreversible manner when the crack opening width increases monotonically. Alternatively, when the crack undergoes unloading/reloading, we verify that the commonly applied coupled and uncoupled unloading/reloading relations produce selfhealing behavior within the formulation. To address this issue, we present a new coupled unloading/reloading relation which maintains the thermodynamic consistency of the PPR model.

\section{Acknowledgments}

We thank the two anonymous reviewers for their critical input which contributed to improve the contents of the manuscript. We acknowledge support from the Natural Sciences and Engineering Research Council of Canada and from the U.S. National Science Foundation (NSF) through grants \#1321661 and \#1437535. We also acknowledge support from the Raymond Allen Jones Chair at the Georgia Institute of Technology. The information presented in this publication is the sole opinion of the authors and does not necessarily reflect the views of the sponsors or sponsoring agencies. 


\section{Nomenclature}

$\alpha$

$\alpha_{v}$

$\bar{\delta}_{n}$

$\bar{\delta}_{t}$

$\beta$

$\beta_{v}$

$\Delta_{n}$

$\delta_{n}$

$\Delta_{n}^{i+1}$

$\Delta_{n}^{i}$

$\Delta_{t}$

$\delta_{t}$

$\Delta_{t}^{i+1}$

$\Delta_{t}^{i}$

$\dot{\Psi}$

$\Gamma_{n}$

$\Gamma_{t}$

$\kappa$

$\kappa_{n}$

$\kappa_{n}^{i+1}$

$\kappa_{n}^{i}$

$\kappa_{t}$

$\kappa_{t}^{i+1}$

$\kappa_{t}^{i}$

$\lambda_{n}$

$\lambda_{t}$

$(\cdot)$

$\langle\cdot\rangle$

$\mathbf{D}^{v}$

$\stackrel{\circ}{w}$

$\phi_{n}$

$\phi_{t}$

$\Psi$ parameter controlling the shape of the cohesive softening curve in the normal direction parameter controlling the shape of the unloading/reloading curve in the normal direction conjugate normal final crack opening width conjugate tangential final crack opening width parameter controlling the shape of the cohesive softening curve in the tangential direction parameter controlling the shape of the unloading/reloading curve in the tangential direction normal separation along the fracture surface normal final crack opening width normal separation along the fracture surface at numerical increment $i+1$

normal separation along the fracture surface at numerical increment $i$

tangential separation along the fracture surface

tangential final crack opening width

tangential separation along the fracture surface at numerical increment $i+1$

tangential separation along the fracture surface at numerical increment $i$

time derivative of the Helmholtz free energy function

energy constant in the PPR model

energy constant in the PPR model

internal history parameter in the coupled unloading/reloading relation

internal history parameter in the normal direction internal history parameter in the normal direction at numerical increment $i+1$

internal history parameter in the normal direction at numerical increment $i$

internal history parameter in the tangential direction

internal history parameter in the tangential direction at numerical increment $i+1$

internal history parameter in the tangential direction at numerical increment $i$

parameter controlling the hardening slope of the PPR model in the normal direction

parameter controlling the hardening slope of the PPR model in the normal direction

time derivative

Macauley bracket

material tangent stiffness matrix of the thermodynamically consistent unloading/reloading relation

stress power

fracture energy in the normal direction (with zero tangential separation)

fracture energy in the tangential direction (with

zero tangential separation)

total Helmholtz free energy function
$\Psi_{i} \quad$ component of the total Helmholtz free energy function

$\Psi_{n} \quad$ Helmholtz free energy functions related to normal separation

$\Psi_{t} \quad$ Helmholtz free energy functions related to tangential separation

time-like parameter

cohesive traction vector

displacement vector

dissipation

scalar damage parameter

normal component of the material tangent stiffness matrix for loading

$D_{n n}^{v}, D_{n t}^{v} \quad$ normal components of the material tangent stiffness matrix for unloading/reloading

$d_{n} \quad$ normal scalar damage parameter

$d_{n}^{(n)} \quad$ normal scalar damage parameter due to normal separation

$d_{n}^{(t)} \quad$ normal scalar damage parameter due to tangential separation

$D_{t n}^{v}, D_{t t}^{v} \quad$ tangential components of the material tangent stiffness matrix for unloading/reloading

$D_{t t} \quad$ tangential component of the material tangent stiffness matrix for loading

$d_{t} \quad$ tangential scalar damage parameter

$d_{t}^{(n)} \quad$ tangential scalar damage parameter due to normal separation

$d_{t}^{(t)} \quad$ tangential scalar damage parameter due to tangential separation

$E_{n} \quad$ initial stiffness parameter in the normal direction

$E_{t} \quad$ initial stiffness parameter in the tangential direction

nondimensional exponent in the PPR model

nondimensional exponent in the PPR model

normal cohesive traction in the original PPR model

tangential cohesive traction in the original PPR model

cohesive traction in the normal direction

normal cohesive traction for the unloading/reloading relation

$T_{t} \quad$ cohesive traction in the tangential direction

$T_{t}^{v} \quad$ tangential cohesive traction for the unloading/reloading relation

\section{Appendix A. Comparison Between the Present Thermo- dynamically Consistent formulation and the Original PPR Model}

Here, the proposed thermodynamically consistent form of the PPR model is compared to the original form of the model, presented in [2], for the case of monotonic loading. First, we present the derivation of the initial stiffness parameters $E_{n}$ and $E_{t}$ in Equations (11) and (12). Next, the damage parameters $d_{n}$ and $d_{t}$ in Equations (18) and (19) are simplified for the case of monotonic loading. Lastly, the proposed thermodynamically consistent model is compared to the original PPR model. The 
result of this investigation illustrates that the proposed thermodynamically consistent form of the PPR model is equivalent to the original model under monotonic loading conditions.

The initial stiffness parameters $E_{n}$ and $E_{t}$, in Equations (11) and (12), are obtained by evaluating $D_{n n}(0,0)$ and $D_{t t}(0,0)$ (from Equation (35) in [18]):

$$
\begin{gathered}
E_{n}=D_{n n}(0,0)=\frac{\Gamma_{n}}{\delta_{n}^{2}}\left[\left(m^{2}-m\right)\left(\frac{m}{\alpha}\right)^{m-2}+\left(\alpha^{2}-\alpha\right)\left(\frac{m}{\alpha}\right)^{m}\right. \\
\left.-2 m \alpha\left(\frac{m}{\alpha}\right)^{m-1}\right]\left[\Gamma_{t}\left(\frac{n}{\beta}\right)^{n}+\left\langle\phi_{t}-\phi_{n}\right\rangle\right] \\
=-\frac{\Gamma_{n}}{\delta_{n}^{2}}\left(\frac{m}{\alpha}\right)^{m-1}(m+\alpha)\left[\Gamma_{t}\left(\frac{n}{\beta}\right)^{n}+\left\langle\phi_{t}-\phi_{n}\right\rangle\right], \quad \\
E_{t}=D_{t t}(0,0)=\frac{\Gamma_{t}}{\delta_{t}^{2}}\left[\left(n^{2}-n\right)\left(\frac{n}{\beta}\right)^{n-2}+\left(\beta^{2}-\beta\right)\left(\frac{n}{\beta}\right)^{n}\right. \\
\left.-2 n \beta\left(\frac{n}{\beta}\right)^{n-1}\right]\left[\Gamma_{n}\left(\frac{m}{\alpha}\right)^{m}+\left\langle\phi_{n}-\phi_{t}\right\rangle\right] \\
=-\frac{\Gamma_{t}}{\delta_{t}^{2}}\left(\frac{n}{\beta}\right)^{n-1}(n+\beta)\left[\Gamma_{n}\left(\frac{m}{\alpha}\right)^{m}+\left\langle\phi_{n}-\phi_{t}\right\rangle\right] .
\end{gathered}
$$

The damage parameters $d_{n}$ and $d_{t}$ in Equations (18) and (19) account for the degradation of the cohesive tractions as the displacement jump increases. Conceptually, the proposed damage parameters $d_{n}$ and $d_{t}$ account for difference between the elastic stresses in the normal and tangential directions and the corresponding inelastic stresses predicted by the original formulation of the model [2]. If monotonic loading is assumed, the internal history parameters become $\kappa_{n}=\Delta_{n}$ and $\kappa_{t}=\left|\Delta_{t}\right|$. Replacing these expressions for $\kappa_{n}$ and $\kappa_{t}$ in Equations (18) and (21), we obtain:

$$
\begin{gathered}
1-d_{n}\left(\Delta_{n},\left|\Delta_{t}\right|\right)=\frac{\Gamma_{n}}{E_{n} \Delta_{n} \delta_{n}}\left[m\left(1-\frac{\Delta_{n}}{\delta_{n}}\right)^{\alpha}\left(\frac{m}{\alpha}+\frac{\Delta_{n}}{\delta_{n}}\right)^{m-1}\right. \\
\left.-\alpha\left(1-\frac{\Delta_{n}}{\delta_{n}}\right)^{\alpha-1}\left(\frac{m}{\alpha}+\frac{\Delta_{n}}{\delta_{n}}\right)^{m}\right] \\
\times\left[\Gamma_{t}\left(1-\frac{\left|\Delta_{t}\right|}{\delta_{t}}\right)^{\beta}\left(\frac{n}{\beta}+\frac{\left|\Delta_{t}\right|}{\delta_{t}}\right)^{n}+\left\langle\phi_{t}-\phi_{n}\right\rangle\right], \\
1-d_{t}\left(\Delta_{n},\left|\Delta_{t}\right|\right)=\frac{\Gamma_{t}}{E_{t}\left|\Delta_{t}\right| \delta_{n}}\left[n\left(1-\frac{\left|\Delta_{t}\right|}{\delta_{t}}\right)^{\beta}\left(\frac{n}{\beta}+\frac{\left|\Delta_{t}\right|}{\delta_{t}}\right)^{n-1}\right. \\
\left.-\beta\left(1-\frac{\left|\Delta_{t}\right|}{\delta_{t}}\right)^{\beta-1}\left(\frac{n}{\beta}+\frac{\left|\Delta_{t}\right|}{\delta_{t}}\right)^{n}\right] \\
\times\left[\Gamma_{n}\left(1-\frac{\Delta_{n}}{\delta_{n}}\right)^{\alpha}\left(\frac{m}{\alpha}+\frac{\Delta_{n}}{\delta_{n}}\right)^{m}+\left\langle\phi_{n}-\phi_{t}\right\rangle\right] .
\end{gathered}
$$

Substituting Equations (A.3), (A.4) and (10) into Equation (7) yields:

$$
\begin{aligned}
T_{n}\left(\Delta_{n}, \Delta_{t}\right)= & \frac{\Gamma_{n}}{\delta_{n}}\left[m\left(1-\frac{\Delta_{n}}{\delta_{n}}\right)^{\alpha}\left(\frac{m}{\alpha}+\frac{\Delta_{n}}{\delta_{n}}\right)^{m-1}\right. \\
& \left.-\alpha\left(1-\frac{\Delta_{n}}{\delta_{n}}\right)^{\alpha-1}\left(\frac{m}{\alpha}+\frac{\Delta_{n}}{\delta_{n}}\right)^{m}\right] \\
\times & {\left[\Gamma_{t}\left(1-\frac{\left|\Delta_{t}\right|}{\delta_{t}}\right)^{\beta}\left(\frac{n}{\beta}+\frac{\left|\Delta_{t}\right|}{\delta_{t}}\right)^{n}+\left\langle\phi_{t}-\phi_{n}\right\rangle\right], } \\
T_{t}\left(\Delta_{n}, \Delta_{t}\right)= & \frac{\Gamma_{t}}{\delta_{t}}\left[n\left(1-\frac{\left|\Delta_{t}\right|}{\delta_{t}}\right)^{\beta}\left(\frac{n}{\beta}+\frac{\left|\Delta_{t}\right|}{\delta_{t}}\right)^{n-1}\right. \\
& \left.-\beta\left(1-\frac{\left|\Delta_{t}\right|}{\delta_{t}}\right)^{\beta-1}\left(\frac{n}{\beta}+\frac{\left|\Delta_{t}\right|}{\delta_{t}}\right)^{n}\right] \\
\times & {\left[\Gamma_{n}\left(1-\frac{\Delta_{n}}{\delta_{n}}\right)^{\alpha}\left(\frac{m}{\alpha}+\frac{\Delta_{n}}{\delta_{n}}\right)^{m}+\left\langle\phi_{n}-\phi_{t}\right\rangle\right] \frac{\Delta_{t}}{\left|\Delta_{t}\right|} . }
\end{aligned}
$$

As expected, these expressions are identical to the cohesive tractions predicted by the original PPR model [2], demonstrating the equivalence between the proposed thermodynamically consistent formulation and the original PPR model formulation under conditions of monotonic loading.

\section{References}

[1] J. Mosler, I. Scheider, A thermodynamically and variationally consistent class of damage-type cohesive models, Journal of the Mechanics and Physics of Solids 59 (2011) 1647-1668.

[2] K. Park, G. H. Paulino, J. R. Roesler, A unified potential-based cohesive model for mixed-mode fracture, Journal of the Mechanics and Physics of Solids 57 (2009) 891-908.

[3] D. W. Spring, G. H. Paulino, Computational homogenization of the debonding of particle reinforced composites: The role of interphases in interfaces, Computational Materials Science 109 (2015) 209-224.

[4] D. W. Spring, Failure processes in soft and quasi-brittle materials with nonhomogeneous microstructures, Ph.D. thesis, University of Illinois at Urbana-Champaign (2015).

[5] F. Cazes, M. Coret, A. Combescure, A. Gravouil, A thermodynamic method for the construction of a cohesive law from a nonlocal damage model, International Journal of Solids and Structures 46 (2009) 14761490.

[6] R. Dimitri, M. Trullo, L. De Lorenzis, G. Zavarise, A consistency assessment of coupled cohesive zone models for mixed-mode debonding problems, Fracture and Structural Integrity 29 (2014) 266-283.

[7] J. P. McGarry, É. Ó. Máirtin, G. E. Beltz, Potential-based and nonpotential-based cohesive zone formulations under mixed-mode separation and over-closure. Part I: Theoretical analysis, Journal of the Mechanics and Physics of Solids 63 (2014) 336-362.

[8] É. Ó. Máirtin, G. Parry, G. E. Beltz, J. P. McGarry, Potential-based and non-potential-based cohesive zone formulations under mixed-mode separation and over-closure. Part II: Finite element applications, Journal of the Mechanics and Physics of Solids 63 (2014) 363-385.

[9] T. C. Gasser, G. A. Holzapfel, Geometrically non-linear and consistently linearized embedded strong discontinuity models for 3D problems with an application to the dissection analysis of soft biological tissues, Computer Methods in Applied Mechanics and Engineering 192 (2003) 5059-5098.

[10] J. Mergheim, P. Steinmann, A geometrically nonlinear FE approach for the simulation of strong and weak discontinuities, Computer Methods in Applied Mechanics and Engineering 195 (2006) 5037-5052.

[11] R. Radulovic, O. T. Bruhns, J. Mosler, Effective 3D failure simulations by combining the advantages of embedded Strong Discontinuity Ap- 
proaches and classical interface elements, Engineering Fracture Mechanics 78 (2011) 2470-2485.

[12] B. D. Coleman, M. E. Gurtin, Thermodynamics with internal state variables, Journal of Chemical Physics 47 (1967) 597-613.

[13] J. Lemaitre, A Course on Damage Mechanics, Springer-Verlag, Berlin, 1992.

[14] J. C. Simo, T. J. R. Hughes, Computational Inelasticity, Springer-Verlag, New York, 1998.

[15] J. Lemaitre, J. L. Chaboche, Mechanics of Solid Materials, Cambridge University Press, 1990.

[16] B. D. Coleman, W. Noll, The thermodynamics of elastic materials with heat conduction and viscosity, Archive for Rational Mechanics and Analysis 13 (1963) 167-178.

[17] K. Park, Potential-based fracture mechanics using cohesive zone and virtual internal bond modeling, Ph.D. thesis, University of Illinois at UrbanaChampaign (2009).

[18] K. Park, G. H. Paulino, Computational implementation of the PPR potential-based cohesive model in Abaqus: Educational perspective, Engineering Fracture Mechanics 93 (2012) 239-262.

[19] D. W. Spring, G. H. Paulino, A growing library of three-dimensional cohesive elements for use in Abaqus, Engineering Fracture Mechanics 126 (2014) 190-216.

[20] A. de Andres, J. Perez, M. Ortiz, Elastoplastic finite element analysis of three-dimensional fatigue crack growth in aluminum shafts subjected to axial loading, International Journal of Solids and Structures 36 (1999) 2231-2258.

[21] O. Nguyen, E. A. Repetto, M. Ortiz, R. A. Radovitzky, A cohesive model of fatigue crack growth, International Journal of Fracture 110 (2001) 351369.

[22] S. Maiti, P. H. Geubelle, A cohesive model for fatigue failure of polymers, Engineering Fracture Mechanics 72 (2005) 691-708. 